\title{
Flux penetration in a superconducting film partially capped with a conducting layer
}

\author{
J. Brisbois,,${ }^{1,}$ V. N. Gladilin,,${ }^{2,3}$ J. Tempere,${ }^{3}$ J. T. Devreese, ${ }^{3}$ V. V. Moshchalkov, ${ }^{2}$ F. Colauto, ${ }^{4}$ M. Motta, ${ }^{4}$ T. H. Johansen,,${ }^{5,6}$ \\ J. Fritzsche, ${ }^{7}$ O.-A. Adami, ${ }^{1}$ N. D. Nguyen,,${ }^{1}$ W. A. Ortiz ${ }^{4}$ R. B. G. Kramer, ${ }^{8,9}$ and A. V. Silhanek ${ }^{1}$ \\ ${ }^{1}$ Experimental Physics of Nanostructured Materials, Q-MAT, CESAM, Université de Liège, B-4000 Sart Tilman, Belgium \\ ${ }^{2}$ INPAC-Institute for Nanoscale Physics and Chemistry, Nanoscale Superconductivity and Magnetism Group, \\ K.U. Leuven, B-3001 Leuven, Belgium \\ ${ }^{3}$ Departement Fysica, Universiteit Antwerpen, B-2020 Antwerpen, Belgium \\ ${ }^{4}$ Departamento de Física, Universidade Federal de São Carlos, 13565-905 São Carlos, SP, Brazil \\ ${ }^{5}$ Department of Physics, University of Oslo, P.O. Box 1048 Blindern, 0316 Oslo, Norway \\ ${ }^{6}$ Institute for Superconducting and Electronic Materials, University of Wollongong, Northfields Avenue, Wollongong, NSW 2522, Australia \\ ${ }^{7}$ Department of Applied Physics, Chalmers University of Technology, S-412 96 Göteborg, Sweden \\ ${ }^{8}$ Université Grenoble Alpes, Institut NEEL, F-38000 Grenoble, France \\ ${ }^{9}$ CNRS, Institut NEEL, F-38000 Grenoble, France
}

(Received 31 August 2016; revised manuscript received 23 December 2016; published 10 March 2017)

\begin{abstract}
The influence of a conducting layer on the magnetic flux penetration in a superconducting $\mathrm{Nb}$ film is studied by magneto-optical imaging. The metallic layer partially covering the superconductor provides an additional velocity-dependent damping mechanism for the flux motion that helps to protect the superconducting state when thermomagnetic instabilities develop. If the flux advances with a velocity slower than $w=2 / \mu_{0} \sigma t$, where $\sigma$ is the cap layer conductivity and $t$ is its thickness, the flux penetration remains unaffected, whereas for incoming flux moving faster than $w$, the metallic layer becomes an active screening shield. When the metallic layer is replaced by a perfect conductor, it is expected that the flux braking effect will occur for all flux velocities. We investigate this effect by studying $\mathrm{Nb}$ samples with a thickness step. Some of the observed features, namely the deflection of the flux trajectories at the border of the thick center, as well as the favored flux penetration at the indentation, are reproduced by time-dependent Ginzburg-Landau simulations.
\end{abstract}

DOI: 10.1103/PhysRevB.95.094506

\section{INTRODUCTION}

It has been shown recently that magnetic flux avalanches triggered in a superconducting film are diverted from their initial trajectory when they encounter a conductive layer deposited on top of the superconductor, but electrically insulated from it [1-4]. This phenomenon arises from the electromagnetic braking of the flux propagation, caused by the eddy currents induced in the conductive layer [5-8]. The question as to whether a single element of the flux front, i.e., a superconducting vortex, could also undergo any deflection of its trajectory when entering in the region covered by a conducting layer has recently been tackled by appealing to a classical analogy, consisting of a magnetic monopole (the vortex) moving in the vicinity of a metallic film [2].

For a conducting layer without borders, Faraday's induction law indicates that a positively charged magnetic monopole moving at a velocity $v$, at a constant distance from the top of the layer, generates a trail of positive and negative images, receding with a velocity given by

$$
w=2 / \mu_{0} \sigma t
$$

where $\sigma$ is the cap layer conductivity and $t$ is its thickness [9-12]. Depending on the ratio $v / w$, two distinct limiting situations appear. For low velocities, $v / w \ll 1$, the trail of negative images dominates over the positive images (see Fig. 3 of Ref. [2]), giving rise to a drag force $\eta v$, where $\eta$ is the damping coefficient, constant in this velocity regime. At high monopole velocities, $v / w \gg 1$, only the positive image

\footnotetext{
*jbrisbois@ulg.ac.be
}

located just below the monopole remains, and the drag force tends to zero, i.e., $\eta \rightarrow 0$. In between these two regimes, the drag force reaches a maximum value at $v / w \sim 1.27$. Considering a 500-nm-thick $\mathrm{Cu}$ layer, $w \sim 60 \mathrm{~m} / \mathrm{s}$ at $10 \mathrm{~K}$, whereas $v$ can be as high as $1000 \mathrm{~m} / \mathrm{s}$ in a Nb film [13], so that flux quanta motion may cross over these two dynamic regimes.

A possible way to reach extreme high flux propagation velocities $v$ consists in triggering flux avalanches [14] or kinematic vortices [15] with typical velocities much higher than those of Abrikosov vortices [16]. Recent experiments have indeed shown that a thick $\mathrm{Cu}$ layer on top of a superconducting Al film leads to changes in the dynamic response as seen in the voltage-current characteristics at high drives [17]. However, in the high-speed regime, the nature of the vortex changes, the temperature rises locally, and the classical analogy brought up in Ref. [2] is no longer strictly valid. An alternative way to explore the high-velocity regime while keeping the Abrikosov vortex structure is to substantially decrease $w$, either by increasing $\sigma$ or $t$. Notice, however, that when $t$ becomes comparable to the skin depth, further increase of the metal thickness will not impact $w$. In this context, it is interesting to consider the limiting case of a conductive layer with infinite conductivity, where the damping contribution of the eddy currents becomes negligible, in opposition to the velocity-dependent case when using a normal metal. The closest way to implement a perfect conductivity layer is to replace the metal by a superconducting film. As originally discussed by Giaever [18], the resulting superconducting bilayer separated by a thin insulating film exhibits electric and magnetic responses similar to those obtained for two films directly short-circuited. This happens because the magnetic coupling between the two collinear pieces of the vortex increases as the thickness of the insulating 


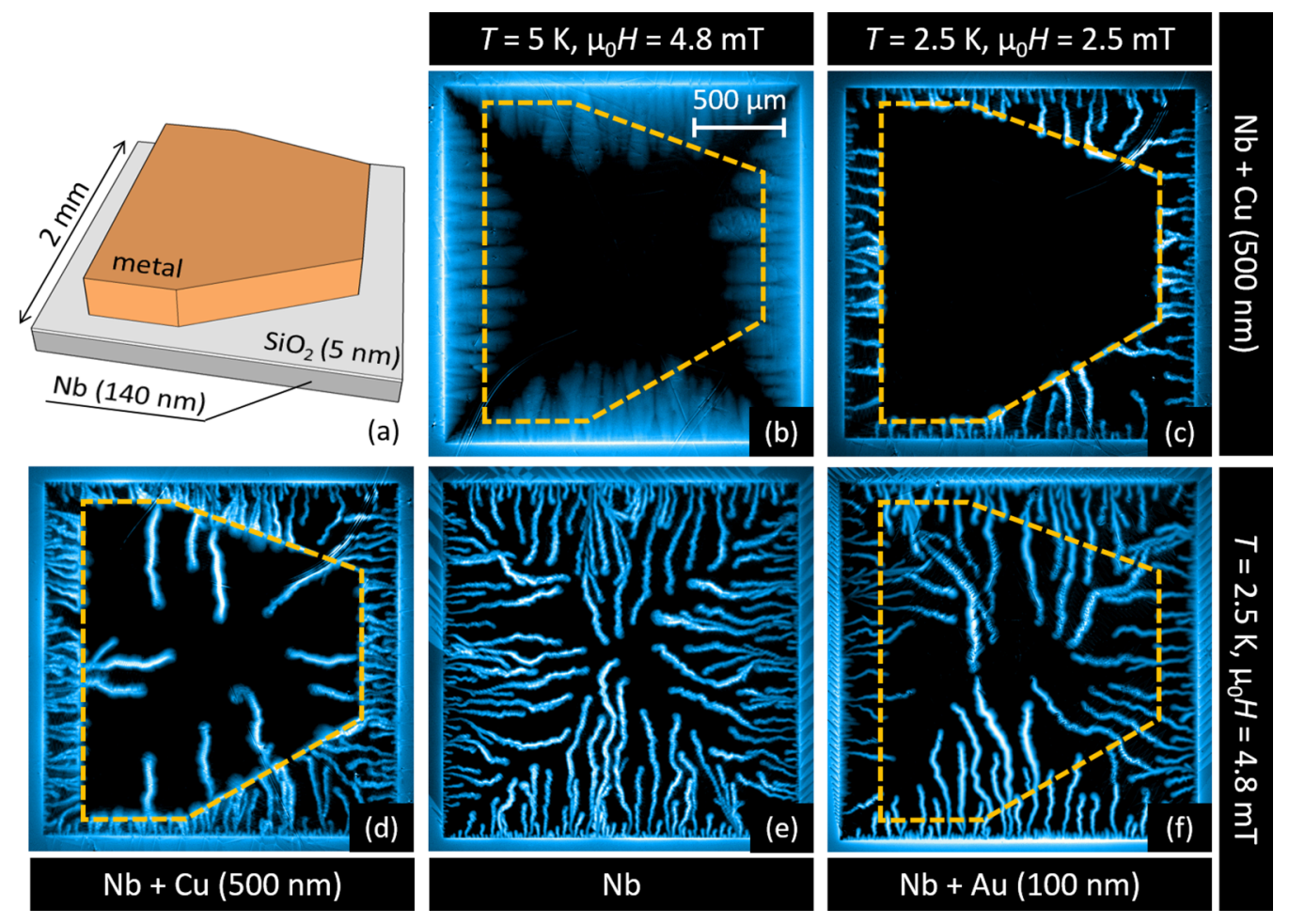

FIG. 1. (a) Sketch of the 140-nm-thick Nb films partially covered by a polygonal metallic layer, either 500-nm-thick Cu [panels (b)-(d)] or 100-nm-thick Au [panel (f)]. All the magneto-optical images were obtained after cooling down the sample before applying an out-of-plane magnetic field (zero-field-cooling procedure). (b) Smooth flux penetration in the $\mathrm{Nb}$ at $T=5 \mathrm{~K}$ and $\mu_{0} H=4.8 \mathrm{mT}$. (c) Shielding of flux avalanches at $T=2.5 \mathrm{~K}$ and $\mu_{0} H=2.5 \mathrm{mT}$. (d) When the magnetic field is increased to $\mu_{0} H=4.8 \mathrm{mT}$, some avalanches are able to overcome the repulsive force from the interface. (e) Flux avalanches in a 140-nm-thick bare Nb film for $\mu_{0} H=4.8 \mathrm{mT}$. (f) Weak shielding of flux avalanches for $\mathrm{Nb}$ partially covered by 100 -nm-thick $\mathrm{Au}$, a system in which the influence of the normal-metal layer on the superconductor can be compared to that of the mirror of the garnet used in MOI.

film decreases. In the limiting case of no insulating layer at all, this corresponds to a superconductor with a step in thickness.

It is precisely the investigation of this limiting case that motivates the present work. To that end, we first extend our previous investigation [2] by exploring the variations of the conductivity and thickness of a metallic layer deposited on top of the superconductor. Interestingly, these measurements suggest that certain precautions need to be taken to ensure that the invasiveness of the magneto-optical imaging (MOI) technique remains at a minimum level [19]. We then address the particular case of very high conductivity, corresponding to a superconducting film with a step in thickness, the central part of the sample being thicker than its borders. As anticipated above, this system can be considered as the superposition of two superconducting films of different size and thickness. The MOI technique shows that at low enough applied fields, the flux front is unable to penetrate into the thicker part of the sample. This effect is further reinforced by the increase of line vortex energy, not considered in the classical model. This behavior contrasts with what is observed in a sample with a thinner center. In the last section, we discuss the flux penetration and interaction with a thickness step at the single vortex level as described by time-dependent Ginzburg-Landau simulations. The present study is also relevant for understanding the flux penetration in superconducting samples with terraces and thickness modulations [20], and it complements early investigations of static flux distributions near surface steps [21,22] and in mesoscopic samples [23].

\section{EXPERIMENTAL DETAILS}

\section{A. Sample fabrication}

The superconducting samples partially covered by a metallic layer are $2 \times 2 \mathrm{~mm}^{2} \mathrm{Nb}$ films with a thickness of $140 \mathrm{~nm}$. They are fabricated on a 2-in. Si wafer by e-beam evaporation in an ultrahigh-vacuum (UHV) system. A sketch of the resulting heterostructure is represented in Fig. 1(a). A 5 -nm-thick $\mathrm{SiO}_{2}$ layer is deposited on top of the $\mathrm{Nb}$ by magnetron sputtering in order to avoid proximity effects between the superconductor and the metal. In a subsequent process step, either a 500-nm-thick copper or a 100-nmthick gold layer of polygonal shape, defined by optical laser lithography, is evaporated on top of the structure. The metallic polygon is placed away from the sample borders so as to permit magnetic flux avalanches to be triggered freely at the borders of the $\mathrm{Nb}$ film. Moreover, this particular shape allows the flux front to reach the metal at different angles of incidence.

The samples with a central thickness step, represented in Figs. 2(a) and 2(e), were fabricated according to the following protocol: a $200-\mathrm{nm}$-thick $\mathrm{Nb}$ film was deposited by dc-sputtering on a 2 -in. Si wafer, with a deposition rate of $0.67 \mathrm{~nm} / \mathrm{s}$ while keeping the substrate at room temperature. It was then coated by a protective photoresist layer before dicing 


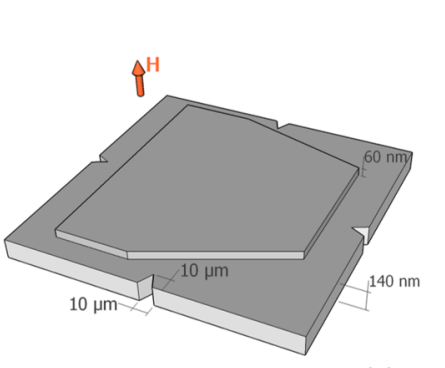

(a)

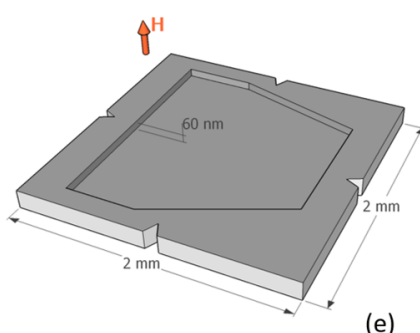

(e)
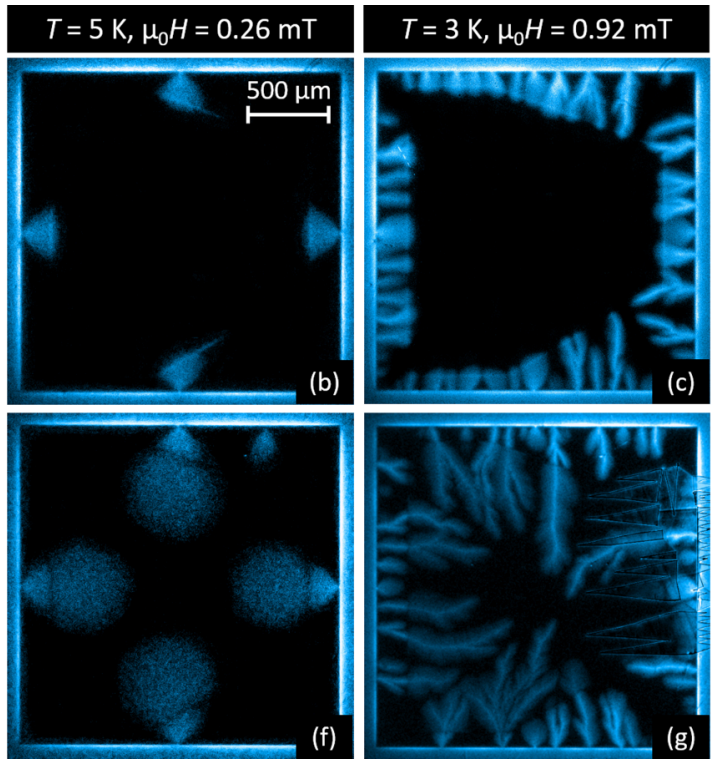
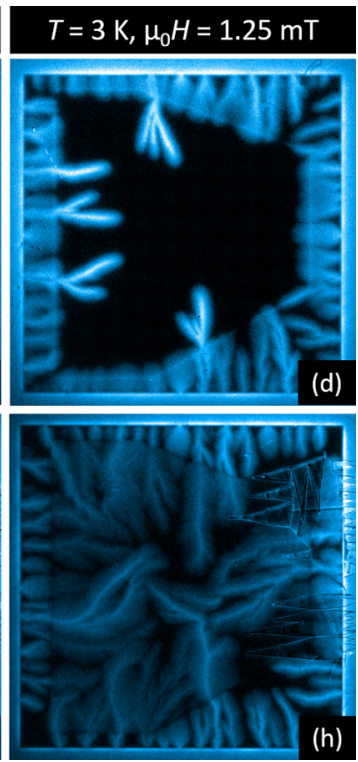

FIG. 2. Panels (a) and (e) show a sketch of the samples layout, consisting of vertically structured Nb thin films with a thicker or thinner center. For the sake of clarity, the illustrations do not respect relative dimension scaling. Magneto-optical images of the magnetic flux penetration into the samples are taken after zero-field cooling. Panels (b)-(d) correspond to the sample with the thick center, whereas panels (f)-(h) show the results for the sample with the thin center. The second column evidences the smooth flux penetration in the critical state regime at $T=5 \mathrm{~K}$. The third and fourth columns show abrupt magnetic flux avalanches at $T=3 \mathrm{~K}$ for two different applied fields.

the substrate to a smaller size $\left(20 \times 30 \mathrm{~mm}^{2}\right)$. After cleaning with acetone, a negative tone photoresist mask was defined, shaping the polygonal central part of the sample. Argon ion milling was performed until the thickness of the uncovered $\mathrm{Nb}$ was reduced down to $140 \mathrm{~nm}$, so they can be readily compared with the samples with a metallic capping layer. To fabricate the thin center samples, an additional step was realized, consisting in the preparation of a second soft mask of positive photoresist defining the central part of the Nb sample. This was followed by an ion milling of the thin center to reduce its thickness to $80 \mathrm{~nm}$. A last soft mask of positive photoresist defining the square shape of the outer rims of the samples was then prepared before etching away the surroundings of the squares with $\mathrm{CF}_{4}$. A final dicing step was performed in order to have individual chips, each carrying one of the cofabricated samples. At the middle of each side of the sample's square outer rim, a $10-\mu \mathrm{m}$-long triangular indentation was lithographically defined in order to facilitate the penetration of magnetic flux through this point [24] and to better visualize the influence of the thickness step. For the sake of comparability, the geometrical shape of the central thickness step has been made identical to that used for the metallic layers. The superconducting properties of the $\mathrm{Nb}$ films have been studied in Ref. [24], giving the transition temperature $T_{\mathrm{c}}=9 \mathrm{~K}$ and the coherence length $\xi=9.7 \mathrm{~nm}$.

\section{B. Magneto-optical imaging (MOI)}

The MOI technique is employed to image the magnetic flux distribution. It relies on the Faraday effect in an indicator film placed on top of the superconducting specimen. A beam of polarized light will have its polarization rotated proportionally to the local value of the magnetic field in the indicator, providing us with bidimensional images of the magnetic field magnitude, where bright (dark) areas correspond to higher (lower) fields [25,26]. The indicators used in the present work are Bi-substituted yttrium iron garnet films (Bi:YIG) with in-plane magnetization, and they are covered with a 100 -nm-thick Al mirror. The images of the magnetic flux distribution were processed using the IMAGEJ and MATLAB softwares, namely to remove the constant background and correct for the inhomogeneous illumination of the sample.

\section{MAGNETIC IMAGING OF FLUX PENETRATION}

To assess the influence of a metallic layer on the superconductor, we first recorded the flux penetration in $\mathrm{Nb}$ films partially covered by a metal (either 500-nm-thick $\mathrm{Cu}$ or 100-nm-thick Au), represented in Fig. 1(a). Figure 1(b) shows the magnetic field in the $\mathrm{Nb}$ film with 500-nm-thick $\mathrm{Cu}$ after cooling down to $5 \mathrm{~K}$ and subsequently applying an out-of-plane field $\mu_{0} H=4.8 \mathrm{mT}$ (zero-field-cooling procedure). From the image, it is clear that the flux penetration is undisturbed by the metal. This can be understood by comparing the velocity $v$ at which the vortices move, typically a few meters per second, with the receding velocity of the images in the copper, $w_{\mathrm{Cu}} \sim 60 \mathrm{~m} / \mathrm{s}$, estimated from Eq. (1) by using for the resistivity the values listed in Ref. [27]. Since $v \ll w$ (low-velocity regime), the braking force is small, and the repulsion force at the metallic layer border, coming from the asymmetry in the eddy currents, is negligible. In contrast, at low temperatures the heat generated by the flux motion cannot be efficiently evacuated [28] and a thermomagnetic instability regime appears, giving rise to an abrupt avalanche-like flux penetration moving at velocities [29] $v \sim 10-100 \mathrm{~km} / \mathrm{s}$. In other words, in this regime $v \gg w$ and a strong inductive response from the metallic layer is expected. This situation is represented in Fig. 1(c), where the temperature has been 
decreased to $2.5 \mathrm{~K}$, before applying $\mu_{0} H=2.5 \mathrm{mT}$. In this case, the repulsive force at the interface tends to its maximum value, and the flux is prevented from entering the region covered by the copper layer. By further increasing the magnetic field up to $\mu_{0} H=4.8 \mathrm{mT}$ [Fig. 1(d)], the flux first accumulates at the border of the metallic layer and then overcomes the repulsive force of inductive origin, thus penetrating the covered area. The comparison with the flux penetration under the same conditions in a bare $\mathrm{Nb}$ film, represented in Fig. 1(e), highlights the efficiency of the copper layer for shielding the central region of the sample. The efficiency of the screening can be evaluated with the parameter $R$, defined as

$$
R=\frac{I_{\text {covered }}}{I_{\text {bare }}}
$$

where $I_{\text {covered }}$ and $I_{\text {bare }}$ correspond to the light intensity in grayscale, averaged over all the pixels lying, respectively, in the part of the sample covered by the polygonal metallic layer and in the bare superconductor. When the screening is perfect, $R$ should be equal to zero. In Fig. 1(c), the noise and the slight penetration at the borders of the $\mathrm{Cu}$ polygon give us a finite but small value $R=0.10 \pm 0.02$. On the other hand, in the absence of a capping layer, as in Fig. 1(e), the ratio is maximum and has a value of $0.80 \pm 0.10$ for $\mu_{0} H=4.8 \mathrm{mT}$ if we consider a fictive polygon of the same dimensions as in the other samples. The efficient screening we observed in Fig. 1(d) gives $R=0.35 \pm 0.05$ in the $\mathrm{Nb}$ film covered by 500 $\mathrm{nm}$-thick $\mathrm{Cu}$, much smaller than the value for the bare $\mathrm{Nb}$. It is interesting to point out here that such a thick $\mathrm{Cu}$ layer on top of the superconductor can increase the effective heat removal coefficient. In this case, it has been predicted [30] that the areal size of the avalanches is reduced when compared with an uncovered superconducting film. However, the case discussed in this paper is somewhat different since first avalanches are triggered from an uncovered rim of the film and only enter later on in a region covered by $\mathrm{Cu}$.

Let us now consider the case in which $w \sim v$. Under this condition, the repulsive force at the interface is smaller than for $v \gg w$, and hence the screening power of the metallic layer will be weakened. A way to increase $w$ consists, according to Eq. (1), in changing the properties of the metallic layer, either by reducing its thickness or increasing its resistivity. Therefore, we used a 100-nm-thick Au layer, having a resistivity [27] between two and eight times bigger than $\mathrm{Cu}$. This gives a corresponding value of $w \sim 3000 \mathrm{~m} / \mathrm{s}$. The magnetic flux distribution for this sample is represented in Fig. 1(f) and shows a weak screening in the area covered by the gold, associated with a ratio $R=0.61 \pm 0.10$, indeed representing a very weak screening power. We can use this result to comment on the largely assumed noninvasiveness of the magneto-optical imaging technique. Usually, the mirror deposited on the garnet is made of $\mathrm{Al}$ or $\mathrm{Au}$ with a typical thickness of $100 \mathrm{~nm}$, similar to the configuration shown in Fig. 1(f). However, unlike in Fig. 1(f), where the metal is in contact with the superconductor, the distance between the mirror in the magneto-optical layer and the sample surface falls normally in the micrometer range, or a few hundred nanometers if the garnet is physically pressed against the sample surface. The mirror has therefore a minor effect on the structure of avalanches and triggering conditions. Nevertheless, as already stated in Ref. [2], special attention needs to be paid for thick mirrors or superconductors deposited in direct contact with the mirror.

As we pointed out above, the maximum screening power of the metallic sheet is obtained in the dynamic regime where $v \gg w$. This was previously illustrated in the $\mathrm{Nb}$-insulator- $\mathrm{Cu}$ trilayer invaded by flux avalanches. In this case, the high speed flux propagation does not actually correspond to a train of individual flux quanta, but rather to the propagation of a normal/superconductor interface. The question as to what extent the same behavior is expected for flux quanta keeping their tubular morphology (i.e., at low $v$ ) could be tackled by making $w$ as low as possible. Equation (1) tells us that this condition can be met by increasing the conductivity of the layer to its upper limit, for instance by using a superconducting film. The final superconductor-insulator-superconductor trilayer will effectively respond as a single superconducting layer with thickness modulation [see Fig. 2(a)] as long as the critical temperature of both superconducting layers is the same and the thickness of the insulator remains substantially smaller than the magnetic penetration depth.

The upper row of Fig. 2 summarizes the results for a $\mathrm{Nb}$ square film covered in the center by a $\mathrm{Nb}$ layer, thus forming a central step. The layout is the same as for the $\mathrm{Nb}$ films with metallic capping, except for $10-\mu \mathrm{m}$-long triangular indentations at the middle of the sample's border, aiming to ease the flux penetration at that particular position. This feature will help us to identify the effect of the thickness step on the flux front propagation. Indeed, as clearly evidenced by the MO images in Fig. 2, the indentations act as flux faucets as a consequence of a combined effect of current crowding and the formation of parabolic discontinuity lines [24]. At low fields, when the sample is in the Meissner state, screening currents running around the sample perimeter are forced to circumvent the triangular indentations, leading to an increase of the streamline density at the vertices of the indentations [31-36]. This locally higher current density favors the penetration of flux quanta through this particular point of the structure.

Figure 2(b) clearly shows that in the smooth flux penetration regime, the parabolic flux penetration is arrested at the border of the step, where the sample thickens from 140 to $200 \mathrm{~nm}$, and is guided along this border (the guidance is particularly prominent for the tilted borders). This effect has two distinct sources: the electromagnetic braking and the penetration barrier at the thickness step, produced by the increase in the vortex core energy. On the one hand, the thickness step leads to a change in the energy $U$ associated with the vortex cores. The vortex-line energy per unit length $\epsilon$ is given by [37]

$$
\epsilon=\frac{\Phi_{0}^{2}}{4 \pi \mu_{0} \lambda^{2}} \ln \left(\frac{\lambda}{\xi}\right)
$$

where $\Phi_{0}$ is the fundamental flux quantum, $\lambda$ is the magnetic penetration depth, and $\xi$ is the coherence length. Since the core energy $U(x)=\epsilon t(x)$ varies across the thickness step $\Delta t=$ $60 \mathrm{~nm}$, there appears a force pushing the vortex toward the thinner regions of the sample:

$$
F_{\text {core }}=-\frac{\partial U}{\partial x} \sim-\frac{\epsilon t\left(x_{2}\right)-\epsilon\left[t\left(x_{2}\right)+\Delta t\right]}{\xi}=\frac{\epsilon \Delta t}{\xi} \approx 375 \mathrm{pN} .
$$


For our calculations, we took $\lambda \sim 100 \mathrm{~nm}[38]$ and $\xi \sim 10 \mathrm{~nm}$ [24], values obtained in samples similar to ours.

On the other hand, the flux deflection can be thought of as an extension of the previously reported results for a $\mathrm{Nb}$ film with a $\mathrm{Cu}$ layer to the case of very high conductivity $(w=0)$, for which an appreciable transverse force at the interface is expected no matter how slow the flux moves. In other words, for the structure under consideration, the condition $v \gg w$ is always satisfied, and therefore, unlike for the $\mathrm{Cu}$ layer, flux deflection is observed also in the smooth field penetration regime (i.e., in the isothermal critical state). Assuming that the vortex field is described by a magnetic monopole with charge $q=2 \mu_{0} \Phi_{0}$, located at a distance $\lambda$ below the surface of the superconductor [39], the repulsive force has a maximum close to the step, where it is given by [2]

$$
F_{\text {eddy }}=-\frac{2 \mu_{0} q^{2}}{27 \pi^{2} \lambda^{2}} \approx 2.6 \mathrm{pN} .
$$

Note that while this force is significantly smaller than $F_{\text {core }}$, it has a much longer range. Indeed, $F_{\text {core }}$ acts on vortices located at distances from the step on the order of $\xi$, while the range of $F_{\text {eddy }}$ is on the order of $\lambda$ (half-width at half-maximum).

Figure 2(c) shows that within the regime of abrupt flux penetration at $T=3 \mathrm{~K}$ for a field $\mu_{0} H=0.92 \mathrm{mT}$, the $\mathrm{Nb}$ overlayer, and likewise the $\mathrm{Cu}$ layer, impedes the flux to enter the region underneath $(R=0.09 \pm 0.02)$. Further increasing the field eventually forces some avalanches to enter into the protected area [Fig. 2(d)], leading to an increased value of $R=0.32 \pm 0.05$.

We would like to stress that caution must be used when thinking of the thick superconducting central area as a perfect conductor. Indeed, there is an additional constant damping force coming from dissipation in the normal cores of the vortices that can never be suppressed, regardless of the velocity. Therefore, as far as damping is concerned, a superconductor will exhibit a different behavior compared to a perfect conductor, since the braking force is always zero in the latter. However, in the case of the deflection of a vortex, considering a perfect conductor or a superconducting capping layer is equivalent, as what matters is the distribution of the eddy/screening currents in the layer. Therefore, the repulsive force felt by a vortex approaching the layer will be the same in both cases. This is true as long as we are not too close to the step, i.e., at distances $\sim \xi$, where the energy associated with the flux lines dominates over the influence of the eddy currents. Replacing the perfect conductor by a normal metal will only decrease the intensity of the repulsive force based on the eddy current's dependence on conductivity.

For the sake of completeness, we have also investigated a sample in which the thickness decreases from 140 to $80 \mathrm{~nm}$ at the central region (lower row of Fig. 2). This could be considered either as the limiting case of a conducting layer covering the rim of the superconductor, or simply as a reduction of the damping in that central area. As evidenced by Fig. 2(f), nothing impedes the flux from invading the central part of the superconductor. In this panel, an unintentional second penetration point can be identified on the top border. This results from a sample imperfection smaller than the nanostructured indentation. We also observe the presence of a dark line in the flux penetration profile, outlining the thickness step. This is caused by the discontinuity in the value of the critical current across the step. In reality, the screening current streamlines - which would otherwise be parallel to the borders of the film - are deformed by the presence of the indentations, and they are forced to circumvent them. As a consequence, nearly parabolic discontinuity lines are formed [24], as seen in Fig. 2(f). Upon an increase of the applied magnetic field, the deformation of the streamlines propagates further inside the film. As they encounter the thickness step and the associated decrease of the thickness-dependent critical current [40], a sudden bend of the lines is in order, expressing the fact that their densities are different on both sides of the step. This local change in the current direction is the cause of the discontinuity lines parallel to the step borders, which are clearly seen in Fig. 2(f).

Figure 2(g) shows the avalanche-like flux penetration at $T=3 \mathrm{~K}$ for a field $\mu_{0} H=0.92 \mathrm{mT}$. As for the smooth penetration, there is no barrier for the flux penetration into the thinner central part. The branches of the avalanches seem to become wider and more blurred in the thinnest region. This effect is caused by the fact that the central region is farther away from the MOI garnet than the rims of the sample, and therefore the stray field emanating from the avalanches is more spread when reaching the garnet.

The large electric fields and the larger traffic of vortices at the border defects should cause the indentations to be preferred nucleation spots for the development of thermomagnetic instabilities [35]. In contrast to that expectation, we do not observe a more frequent occurrence of thermal flux avalanches at the indentations, but rather the opposite (i.e., avalanches avoid the indentation), confirming a recent experimental report [24]. An explanation for these counterintuitive results is still lacking.

In the pioneering work of Wertheimer and Gilchrist [14], it was shown that the speed of flux jumps in $\mathrm{Nb}$ decreases with increasing thickness and increasing normal state conductivity. This behavior was explained in terms of speed limitation by eddy currents. In our study, since the rim of the samples with thinner and thicker centers always has the same thickness, the avalanches are triggered with similar velocities. However, since the thickness is increased in the central part of the thick center sample, the avalanches tend to decelerate their motion, while for the thin center, the thickness decreases and thus they accelerate. Recently, Vestgården and co-authors [41] showed numerically that the threshold field $H_{\text {th }}$ needed to trigger avalanches increases linearly with sample thickness. This was also confirmed by experimental studies [42], and avalanches become straighter with fewer and thicker branches. This seems to be in agreement with the experimental observation reported here.

\section{TIME-DEPENDENT GINZBURG-LANDAU SIMULATIONS}

In view of the fact that the reported magneto-optical investigation reflects, in a macroscopic scale, the interaction of vortices with the thickness modulation, it is interesting to review this phenomenon at the individual vortex level. Toward that end, we provide simulations based on the timedependent Ginzburg-Landau (TDGL) equations for a sample 
with triangular border indentations and having a polygonalshaped thinner or thicker center, as in the experiments. For the sake of keeping the computation time within reasonable limits, the simulated sample size is scaled down to a $2 \mu \mathrm{m} \times 2 \mu \mathrm{m}$ square. A similar system was recently addressed numerically by Barba-Ortega and co-workers [43]. The coherence length $\xi$ is $16 \mathrm{~nm}$ and the (bulk) penetration depth $\lambda$ is $120 \mathrm{~nm}$. Therefore, the simulations can still be compared with our experimental results, since $\lambda$ and $\xi$ are much smaller than the sample dimensions.

An effectively two-dimensional TDGL equation for the order parameter $\psi$, normalized to 1 and averaged over the inhomogeneous thickness $d(x, y)$ of the superconductor, can be written as $[44,45]$

$$
\begin{aligned}
\left(\frac{\partial}{\partial t}+i \varphi\right) \psi= & \frac{1}{d}\left(\nabla_{2}-i \mathbf{A}\right) d\left(\nabla_{2}-i \mathbf{A}\right) \psi \\
& +2 \psi\left(1-|\psi|^{2}\right) .
\end{aligned}
$$

Here, $\varphi$ and $\mathbf{A}$ are the scalar and vector potentials, respectively, averaged over the superconductor thickness, and $\nabla_{2}=$ $\mathbf{e}_{x} \partial / \partial x+\mathbf{e}_{y} \partial / \partial y$. All the relevant quantities are made dimensionless by expressing lengths in units of $\sqrt{2} \xi$, time in units of $\pi \hbar /\left[4 k_{\mathrm{B}}\left(T_{\mathrm{c}}-T\right)\right]$, magnetic field in units of $\Phi_{0} /\left(4 \pi \xi^{2}\right)=$ $\mu_{0} H_{\mathrm{c} 2} / 2$, current density in units of $\Phi_{0} /\left(2 \sqrt{2} \pi \mu_{0} \lambda^{2} \xi\right)$, and scalar potential in units of $2 k_{\mathrm{B}}\left(T_{\mathrm{c}}-T\right) /(\pi e)$. Here, $\Phi_{0}=$ $\pi \hbar / e$ is the magnetic flux quantum, $\mu_{0}$ is the vacuum permeability, and $H_{\mathrm{c} 2}$ is the second critical field.

The distribution of the scalar potential $\varphi$ is determined from the condition $\boldsymbol{\nabla} \cdot \mathbf{j}=\mathbf{0}$, which reflects the continuity of currents in the superconductor. The total current density $\mathbf{j}$ is given by the sum of the normal and superconducting components:

$$
\begin{gathered}
\mathbf{j}=\mathbf{j}_{\mathrm{n}}+\mathbf{j}_{\mathrm{s}}, \\
\mathbf{j}_{\mathrm{n}}=-\frac{\sigma}{2}\left(\nabla \varphi+\frac{\partial \mathbf{A}}{\partial t}\right), \\
\mathbf{j}_{\mathrm{s}}=\operatorname{Im}\left(\psi^{*} \nabla \psi\right)-\mathbf{A}|\psi|^{2},
\end{gathered}
$$

where $\sigma$ is the normal-state conductivity, which is taken as $\sigma=1 / 12$ in our units [46]. For a superconductor with varying thickness, the aforementioned condition of current continuity can be expressed [44] in a two-dimensional (2D) form:

$$
\frac{\sigma}{2} \nabla_{2}\left(d \nabla_{2} \varphi\right)=\nabla_{2}\left(\mathbf{j}_{\mathrm{s}} d\right) .
$$

The averaged vector potential $\mathbf{A}$ that enters in Eq. (6) can be represented as

$$
\mathbf{A}=\mathbf{A}_{\mathrm{e}}+\mathbf{A}_{\mathrm{s}} .
$$

Here the contribution $\mathbf{A}_{\mathrm{e}}$ corresponds to the externally applied magnetic field $\mathbf{B}_{0}$, while $\mathbf{A}_{\text {s }}$ describes the averaged magnetic field, which is induced in the superconductor by the currents given by Eqs. (7)-(9). For a superconducting layer with inhomogeneous thickness, the latter contribution takes the form

$$
\mathbf{A}_{\mathrm{s}}\left(\mathbf{r}_{2}, t\right)=\frac{1}{2 \pi \kappa^{2}} \int d \mathbf{r}_{2}^{\prime} K\left(\mathbf{r}_{2}, \mathbf{r}_{2}^{\prime}\right) d\left(\mathbf{r}_{2}^{\prime}\right) \mathbf{j}\left(\mathbf{r}_{2}^{\prime}, t\right),
$$

where $\kappa=\lambda / \xi$ is the Ginzburg-Landau parameter and $\mathbf{r}_{2}=$ $\mathbf{e}_{x} x+\mathbf{e}_{y} y$ is the in-plane radius vector. The time-independent symmetric kernel

$$
\begin{aligned}
K\left(\mathbf{r}_{2}, \mathbf{r}_{2}^{\prime}\right)= & F\left(d\left(\mathbf{r}_{2}\right), d\left(\mathbf{r}_{2}^{\prime}\right),\left|\mathbf{r}_{2}-\mathbf{r}_{2}^{\prime}\right|\right) \\
& +F\left(d\left(\mathbf{r}_{2}^{\prime}\right), d\left(\mathbf{r}_{2}\right),\left|\mathbf{r}_{2}-\mathbf{r}_{2}^{\prime}\right|\right)
\end{aligned}
$$

is expressed through the function

$$
\begin{aligned}
F\left(d, d^{\prime}, D\right)= & \frac{1}{d}\left\{\ln \left(\frac{R(d, D)}{R\left(d^{\prime}-d, D\right)}\right)+\frac{1}{2 d^{\prime}}[R(0, D)\right. \\
& \left.\left.+R\left(d^{\prime}-d, D\right)-R\left(d^{\prime}, D\right)-R(-d, D)\right]\right\}
\end{aligned}
$$

with

$$
R(d, D)=d+\sqrt{d^{2}+D^{2}} .
$$

Equation (6) with the scalar and vector potentials defined by Eqs. (10)-(12) and the superconductor-insulator boundary conditions, which assure zero values for both the superconducting and normal components of the current across the boundary, is solved numerically using the approach described in Refs. [47,48].

The results are shown in Fig. 3. For all quantities represented in the images, red corresponds to the highest values while blue corresponds to the lowest values. The first column shows the thickness distribution in the sample: the outer part is $140 \mathrm{~nm}$ thick, while the central part is $200 \mathrm{~nm}$ thick [Fig. 3(a)] and $80 \mathrm{~nm}$ [Fig. 3(e)]. We start with a vortex-free state at zero magnetic field, then a field of $0.06 H_{\mathrm{c} 2}$ is applied, so that penetration of vortices is initiated. The simulations continue until a (meta)stable vortex state is reached. The second column [Figs. 3(b) and 3(f)] shows the squared superconducting order parameter $|\psi|^{2}$, whereas the third column [Figs. 3(c) and 3(g)] corresponds to the self-field in the reached (meta)stable state. The upper images evidence the fact that the thickness step acts as a barrier for the incoming vortices, while nothing impedes the vortex motion toward the center in the thin-center sample. The last column [Figs. 3(d) and 3(h)] represents the trajectories of the vortices captured by the parameter $S(x, y)$, which is defined [49] as the root mean square of the rate of changes in the local Cooper pair density:

$$
S(x, y)=\sqrt{\frac{1}{t_{2}-t_{1}} \int_{t_{1}}^{t_{2}} d t\left(\frac{\partial|\psi(x, y)|^{2}}{\partial t}\right)^{2}},
$$

where $t$ is the time variable and $t_{2}-t_{1}$ is the time integration interval. The parameter $S$ at a given point $(x, y)$ strongly increases when this point is passed by a moving vortex core. Figures 3(d) and 3(h) demonstrate that the indentations at the superconductor edges act as flux taps and favor the penetration of vortices, as is also clearly visible in our experimental results shown in Fig. 2. Interestingly, the branching of trajectories observed in the experiments is also featured in the simulations, as the $S$ parameter shows that vortices take different trajectories when they cross the thickness step, leading to the formation of several paths for flux penetration. It is worth emphasizing that the physics of individual vortices differs substantially from that of flux avalanches, and therefore particular care has to be exerted when drawing analogies between them. 


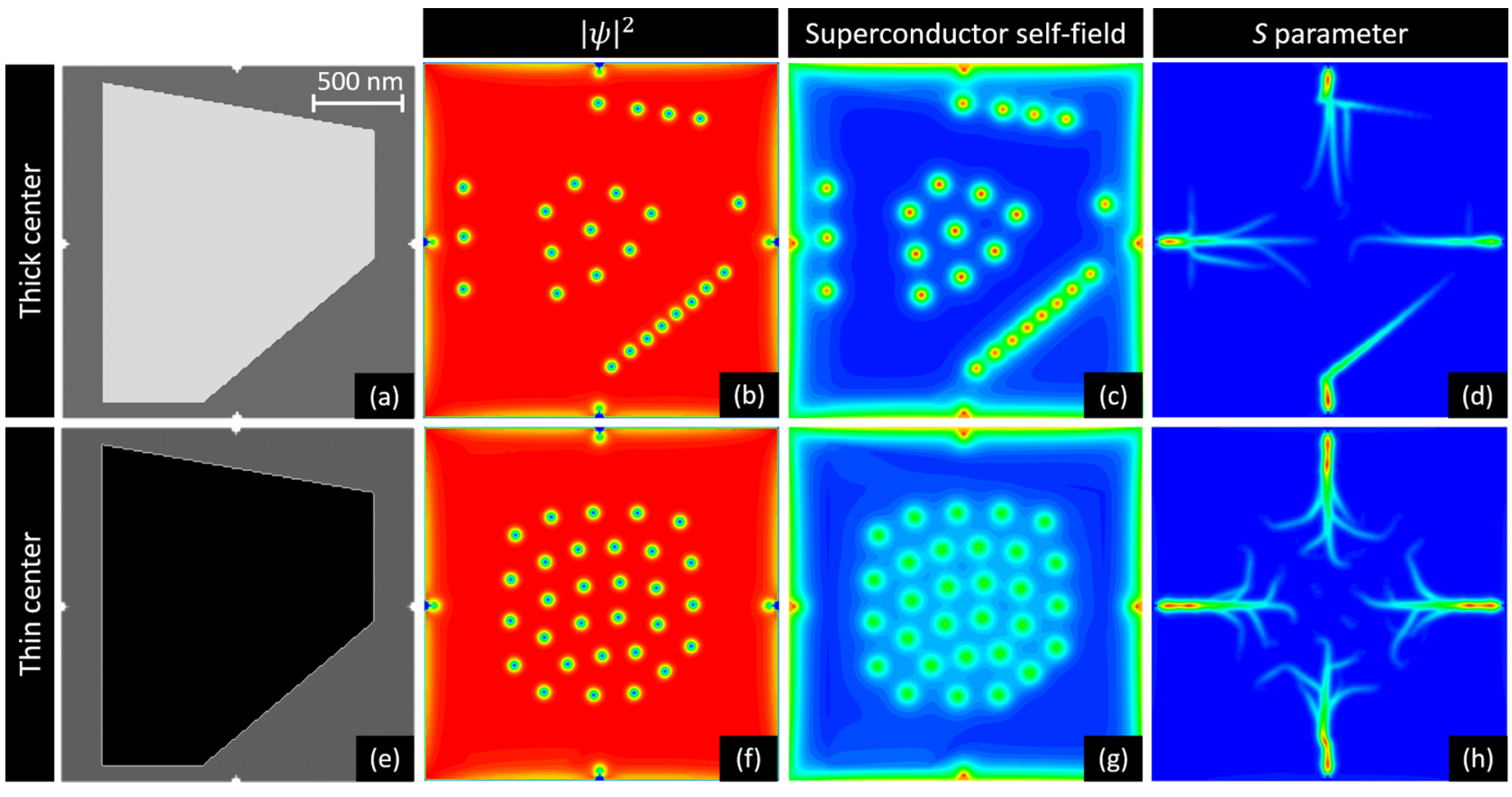

FIG. 3. Results of the time-dependent Ginzburg-Landau simulations on a $2 \times 2 \mu \mathrm{m}^{2} \mathrm{Nb}$ film with a step in thickness, from $140 \mathrm{~nm}$ in the peripheral region to $200 \mathrm{~nm}$ (upper row) or $80 \mathrm{~nm}$ (lower row) in the central region. For each of the plotted quantities, blue, green, and red correspond to low, intermediate, and high values, respectively. The first column shows the distribution of the superconductor thickness. The second column displays the squared modulus of the superconducting order parameter $|\psi|^{2}$ once the system reached a (meta)stable state. The third column is the mapping of the superconductor self-field in the reached (meta)stable state. The last column illustrates the $S$ parameter, representing the trace of the vortex trajectories on the time interval from switching on the field until reaching a stable vortex configuration.

\section{CONCLUSION}

In summary, we have used magneto-optical imaging to characterize the effect of a conductive layer on the magnetic flux propagation in a superconductor, for the smooth penetration as well as for the thermomagnetic instabilities regime. Magnetic flux moving at a velocity $v$ in the vicinity of a conductor induces eddy currents, which in turn generate (i) a force repelling the flux from the border of the conductor, due the asymmetry of the current distribution there, and (ii) a braking force slowing down the flux propagation inside the conductor. When $v \gg w=2 / \mu_{0} \sigma t$, as in the $\mathrm{Nb}$ film partially covered by $500-\mathrm{nm}$-thick copper, magneto-optical images show the clear deflection of flux avalanches, since the repulsive force is nearly maximal in this regime. However, when the $\mathrm{Nb}$ is covered by 100-nm-thick gold, $v \sim w$ and the repulsive force is strongly weakened, leading to weak shielding of the flux from the central part. These measurements show that magneto-optical imaging can be considered as a noninvasive technique as long as the distance between the indicator mirror and the sample surface lies in the micrometer range. In all cases, the metallic layer is inefficient to shield the flux in the smooth penetration regime, where $v \ll w$. A way to keep a maximum repulsive force, even in the low velocities regime, is to replace the metallic layer by a superconductor $(w \approx 0)$. This situation has been approached using $\mathrm{Nb}$ films with a central step in thickness. The repulsive force is present at all flux velocities in the thick center samples, unlike in normal metals. However, in contrast to perfect conductors, there is a constant braking force in superconductors coming from the dissipation in the normal vortex cores, as well as a repulsive force in the vicinity of the border, coming from the vortex core energy. As expected, samples with a thin center area do not impede the flux penetration, but rather feature some blurring of the flux front in the central part. Time-dependent Ginzburg-Landau simulations performed for a smaller sample reproduce the features we highlighted experimentally, including the deflection and the branching of flux trajectories at the thickness step in the thick center sample. Moreover, these simulations confirm the role played by the indentations in the flux penetration, lowering the barrier for vortex entry.

\section{ACKNOWLEDGMENTS}

This work was partially supported by the Fonds de la Recherche Scientifique-FNRS, the ARC Grant No. 13/18-08 for Concerted Research Actions, financed by the WalloniaBrussels Federation, the Brazilian National Council for Scientific and Technological Development (CNPq) and the São Paulo Research Foundation (FAPESP), the program for scientific cooperation F.R.S.-FNRS-CNPq, the Methusalem funding by the Flemish government and the Flemish Science Foundation (FWO), and the COST MP1201 NanoSC Action. J.B. acknowledges support from FRS-FNRS (Research Fellowship). The work of A.V.S. is partially supported by "Mandat d'Impulsion Scientifique" MIS F.4527.13 of the F.R.S.-FNRS. J.T. also acknowledges support from the Research Council of Antwerp University (BOF). The 
work at KU Leuven is supported by the Methusalem Funding by the Flemish government. The LANEF framework (ANR-10-LABX-51-01) and the Nanoscience Foundation are acknowledged for their support with mutualized infrastructure. We would also like to thank C. C. Souza Silva for useful discussions.
[1] J. Albrecht, A. T. Matveev, M. Djupmyr, G. Schütz, B. Stuhlhofer, and H. U. Habermeier, Appl. Phys. Lett. 87, 182501 (2005).

[2] J. Brisbois, B. Vanderheyden, F. Colauto, M. Motta, W. A. Ortiz, J. Fritzsche, N. D. Nguyen, B. Hackens, O.-A. Adami, and A. V. Silhanek, New J. Phys. 16, 103003 (2014).

[3] P. Mikheenko, T. H. Johansen, S. Chaudhuri, I. J. Maasilta, and Y. M. Galperin, Phys. Rev. B 91, 060507 (2015).

[4] P. Mikheenko, J. I. Vestgården, S. Chaudhuri, I. J. Maasilta, Y. M. Galperin, and T. H. Johansen, AIP Adv. 6, 035304 (2016).

[5] R. B. Harrison, J. P. Pendrys, and L. S. Wright, J. Low Temp. Phys. 18, 113 (1975).

[6] M. Danckwerts, A. R. Goñi, C. Thomsen, K. Eberl, and A. G. Rojo, Phys. Rev. Lett. 84, 3702 (2000).

[7] J. Baker and A. G. Rojo, Phys. Rev. B 64, 014513 (2001).

[8] F. Colauto, E. Choi, J. Y. Lee, S. I. Lee, E. J. Patiño, M. G. Blamire, T. H. Johansen, and W. A. Ortiz, Appl. Phys. Lett. 96, 092512 (2010).

[9] J. R. Reitz, J. Appl. Phys. 41, 2067 (1970).

[10] W. M. Saslow, Am. J. Phys. 60, 693 (1991).

[11] W. M. Saslow, Am. J. Phys. 59, 16 (1991).

[12] T. D. Rossing and J. R. Hull, Phys. Teach. 29, 552 (1991).

[13] G. Grimaldi, A. Leo, A. Nigro, S. Pace, and R. P. Huebener, Phys. Rev. B 80, 144521 (2009).

[14] M. R. Wertheimer and J. le G. Gilchrist, J. Phys. Chem. Solids 28, 2509 (1967).

[15] A. Andronov, I. Gordion, V. Kurin, I. Nefedov, and I. Shereshevsky, Physica C 213, 193 (1993).

[16] A. G. Sivakov, A. M. Glukhov, A. N. Omelyanchouk, Y. Koval, P. Müller, and A. V. Ustinov, Phys. Rev. Lett. 91, 267001 (2003).

[17] O.-A. Adami, Z. L. Jelić, C. Xue, M. Abdel-Hafiez, B. Hackens, V. V. Moshchalkov, M. V. Milosević, J. Van de Vondel, and A. V. Silhanek, Phys. Rev. B 92, 134506 (2015).

[18] I. Giaever, Phys. Rev. Lett. 15, 825 (1965).

[19] P. E. Goa, H. Hauglin, A. A. F. Olsen, D. Shantsev, and T. H. Johansen, Appl. Phys. Lett. 82, 79 (2003).

[20] Th. Schuster, M. R. Koblischka, H. Kuhn, H. Kronmüller, G. Friedl, B. Roas, and L. Schultz, Appl. Phys. Lett. 62, 768 (1993).

[21] B. L. T. Plourde, D. J. Van Harlingen, N. Saha, R. Besseling, M. B. S. Hesselberth, and P. H. Kes, Phys. Rev. B 66, 054529 (2002).

[22] F. Pardo, F. de la Cruz, P. L. Gammel, E. Bucher, C. Ogelsby, and D. J. Bishop, Phys. Rev. Lett. 79, 1369 (1997).

[23] J. Barba-Ortega, J. D. Gonzalez, and E. Sardella, J. Low Temp. Phys. 174, 96 (2014).

[24] J. Brisbois, O.-A. Adami, J. I. Avila, M. Motta, W. A. Ortiz, N. D. Nguyen, P. Vanderbemden, B. Vanderheyden, R. B. G. Kramer, and A. V. Silhanek, Phys. Rev. B 93, 054521 (2016).

[25] M. R. Koblischka and R. J. Wijngaarden, Supercond. Sci. Technol. 8, 199 (1995).
[26] Ch. Jooss, J. Albrecht, H. Kuhn, S. Leonhardt, and H. Kronmüller, Rep. Prog. Phys. 65, 651 (2002).

[27] R. A. Matula, J. Phys. Chem. Ref. Data 8, 1147 (1979).

[28] R. G. Mints and A. L. Rahkmanov, Rev. Mod. Phys. 53, 551 (1981).

[29] See Fig. 6(b) in U. Bolz, B. Biehler, D. Schmidt, B.-U. Runge, and P. Leiderer, Europhys. Lett. 64, 517 (2003).

[30] J. I. Vestgarden, D. V. Shantsev, Y. M. Galperin, and T. H. Johansen, Supercond. Sci. Technol. 26, 055012 (2013).

[31] J. R. Clem and K. K. Berggren, Phys. Rev. B 84, 174510 (2011).

[32] O.-A. Adami, D. Cerbu, D. Cabosart, M. Motta, J. Cuppens, W. A. Ortiz, V. V. Moshchalkov, B. Hackens, R. Delamare, J. Van de Vondel, and A. V. Silhanek, Appl. Phys. Lett. 102, 052603 (2013).

[33] D. Cerbu, V. N. Gladilin, J. Cuppens, J. Fritzsche, J. Tempere, J. T. Devreese, V. V. Moshchalkov, A. V. Silhanek, and J. Van de Vondel, New J. Phys. 15, 063022 (2013).

[34] G. Via, C. Navau, and A. Sanchez, J. Appl. Phys. 113, 093905 (2013).

[35] J. I. Vestgården, D. V. Shantsev, Y. M. Galperin, and T. H. Johansen, Phys. Rev. B 76, 174509 (2007).

[36] A. Gurevich and M. Friesen, Phys. Rev. B 62, 4004 (2000).

[37] M. Tinkham, Introduction to Superconductivity, 2nd ed. (Dover, New York, 2004), pp. 152 and 153.

[38] A. I. Gubin, K. S. Il'in, S. A. Vitusevich, M. Siegel, and N. Klein, Phys. Rev. B 72, 064503 (2005).

[39] A. M. Chang, H. D. Hallen, L. Harriott, H. F. Hess, H. L. Kao, J. Kwo, R. E. Miller, R. Wolfe, J. van der Ziel, and T. Y. Chang, Appl. Phys. Lett. 61, 1974 (1992).

[40] F. Hengstberger, M. Eisterer, and H. W. Weber, Appl. Phys. Lett. 96, 022508 (2010).

[41] J. I. Vestgården, Y. M. Galperin, and T. H. Johansen, arXiv:1309.6463.

[42] F. Colauto, E. J. Patiño, M. Aprilli, and W. A. Ortiz, J. Phys.: Conf. Ser. 150, 052038 (2009).

[43] J. Barba-Ortega, E. Sardella, and J. A. Aguiar, Phys. Lett. A 379, 732 (2015).

[44] S. J. Chapman, Q. Du, and M. D. Gunzburger, Z. Angew. Math. Phys. 47, 410 (1996).

[45] V. N. Gladilin, J. Ge, J. Gutierrez, M. Timmermans, J. Van de Vondel, J. Tempere, J. T. Devreese, and V. V. Moshchalkov, New J. Phys. 17, 063032 (2015).

[46] R. Kato, Y. Enomoto, and S. Maekawa, Phys. Rev. B 44, 6916 (1991).

[47] A. V. Silhanek, V. N. Gladilin, J. Van de Vondel, B. Raes, G. W. Ataklti, W. Gillijns, J. Tempere, J. T. Devreese, and V. V. Moshchalkov, Supercond. Sci. Technol. 24, 024007 (2011).

[48] V. N. Gladilin, J. Tempere, J. T. Devreese, and V. V. Moshchalkov, Solid State Commun. 152, 1781 (2012).

[49] V. N. Gladilin, J. Tempere, J. T. Devreese, W. Gillijns, and V. V. Moshchalkov, Phys. Rev. B 80, 054503 (2009). 